\title{
On Short Video in College English Class from the Perspective of Multi-modal Teaching Model
}

\author{
Xin Xiong ${ }^{1}$ and Zhen $\mathrm{Shi}^{1{ }^{1, *}}$ \\ ${ }^{1}$ Foreign Language Studies, Guangxi University of Science \& Technology, Liuzhou, Guangxi 545006, China \\ * Corresponding author. Email: 1340491029@qq.com
}

\begin{abstract}
The popularity of the Internet, especially the rapid rise of self-media, has brought people closer to each other, and at the same time they have a new way of obtaining information and communicating ideas, such as Tiktok, Miaopai, Kuaishou and other short video apps. They are famous of them and popular with people. To explore effective multi-modal teaching models in English classroom of college school, this article analyzes the current information receiving methods and characteristics that young people like to see and hear, combining the individual characteristics of students in language acquiring.
\end{abstract}

Keywords: multi-modal; English in college school; short video; classroom teaching strategies

\section{INTRODUCTION}

The rapid development of network technology has enabled more and more convenient contacts and exchanges between different countries and people. The more open China should also make use of convenient network resources to learn international advanced science and technology, teaching models and teaching ideas. In international spread and communication, there is always differences between language and culture. In order to carry out the communication smoothly and deeply, the education and learning of the international language - English has become the first problem to be solved. At present, many universities also regard passing CET-4 and CET-6 as the requirement students must meet when they graduate. Recent years, Tiktok, Miaopai and Kuaishou on the Internet have brought a new challenge to English teaching and learning. Therefore, it is particularly important to make full use of the characteristics of this short video learning software, combined with the personalized characteristics of students' language learning, to discuss the teaching effect in College English classroom from the multi-modal perspective of English teaching, so as to effectively improve students' English application ability.

\section{MULTI-MODAL TEACHING MODE}

\subsection{Concept of Multi-modal}

In our daily communication, the meaning of discourse is no longer reflected by the single language, but also conveyed by many other nonverbal factors, such as the language environment, the speaker's tone, mood, expression, action and so on. This way of communication does not use a single sense. Short videos use vision and hearing together, which is multi-modal discourse

Multi-modal discourse refers to the phenomenon of using hearing, vision, touch and other senses to communicate through language, image, sound, action and other means and symbolic resources[1]. Speaking of this, people can't help thinking of the media. The two are different concepts. Modal is a means or symbol, and the media is the carrier that realizes these modalities. Modality is a symbol system, and media is the material means of symbol distribution and imprinting (Levine, P. \&amp; Scollon, R., 2004:125)[2] For example, vision, hearing, touch, taste and smell are different modes of our perception of the world, while eyes, ears, hands, tongues and noses are the media to carry the output of these modes.

\subsection{Construction Significance of Multi-modal Teaching Mode}

The theoretical framework of multi-modal discourse research is Halliday's systemic functional linguistics. He believes that language has three "metafunctions", namely ideational metafunction, interpersonal metafunction and textual metafunction. In this context, Kress \&Van Leeuwen put forward the theory of visual grammar in Reading images, further extended Halliday's metafunction into a social symbol, and established "reproduction meaning", "interactive meaning" and "composition meaning" [3].

Multi-modal collaborative reproduction of knowledge. Reproducing meaning refers to restoring the character or event itself through a variety of symbols. The teacher tells the knowledge points by recording a short video. The subtitles in the video appear synchronously with the teacher's explanation, and the colors and fonts are marked and changed according to the key points of the explanation; the use of images plays an auxiliary role in the understanding of the explanation content. The sound 
mainly come from teacher and background music to grasp people's attention from hearing.

Multi-modal discourse enhances interaction. Interactive meaning refers to the discussion between the interpreter in the video and the viewer. When making video, in order to increase the interest of knowledge and mobilize the viewer's emotion, the interpreter needs to guide the viewer to think through rich facial expressions, vivid body movements and cadence of tone.

Different meanings of modal distribution. Kress, G \& Van Leeuwen believes that the combination of multiple modalities in a certain way can form a new whole with new meaning, which is called composition meaning, including information value, information prominence and information framework [3]. In order to reflect the core of different information, the content in the middle of the screen can be adjusted as needed. For example, when the video begins to introduce the content, the teacher, as the problem sender, can appear in the middle of the screen; when entering the topic to explain knowledge, the teacher exits the screen and only sounds appear. The intellectual content is placed in the middle and the subtitles are placed below, so as to reflect the authenticity of information value. In terms of information prominence, it is usually adjusted through the change of color brightness and background, so as to highlight the focus of the content.

\section{DEVELOPMENT STATUS AND POPULAR REASONS OF SHORT VIDEO}

As a new way of communication, short video can contain pictures, words, music and animation. It is a multi-modal presentation loved by the public. Words and pictures transmit information, and the rendering of music can virtually affect the viewer's mood, so as to improve the communication effect, which can not be achieved by any other single communication method

According to the latest statistics of China Internet Network Information Center (CNNIC, September 29, 2020)[4], by June 2020, the number of mobile Internet users in China had reached 932 million, including 818 million short video users, accounting for $87 \%$ of the total Internet users. Among the various short video currently, the Tiktok APP has developed particularly rapidly. As of August 2020, the monthly active users of Tiktok exceeded 600 million; in December, the average number of daily searches on the Tiktok app exceeded 400 million (Xinhuanet, 2021-0105)[5]. The above data fully demonstrates that in the current new media era, people using Tiktok APP to obtain information and record life has become a new trend.

Compared with the traditional paper media, news website, WeChat official account, the information dissemination transmission way of short video is more suitable for people to acquire information through relaxed, pleasant, vivid and flexible visual, auditory and other multi-modal forms. While catching the attention of the audience with its distinctive audio and video, short video can more clearly convey the communication purpose and intention of the information. Static articles usually require the audience to concentrate on reading without distractions in order to fully understand, while short videos can enable the audience to obtain information in a relatively relaxed and pleasant environment, which leads to the fact that the short video information communication mode is more popular than the traditional mode.

\section{INDIVIDUALIZED ANALYSIS OF COLLEGE STUDENTS' ENGLISH LEARNING}

College English learning has an irreplaceable role for their future work, so at this stage it is necessary to fully understand the characteristics of students' study and life for finding efficient teaching ways.

\subsection{Increased Difficulty of Knowledge}

Upon entering the university, students begin to learn professional knowledge which will focus on students' comprehensive application ability. Overall, the number of words that need to be memorized in English learning has increased significantly, and they will face various English situations. Especially for students who do not understand abstract grammar, the single way of explanation will not only make students understand it, but also tend to be afraid of learning English.

Learning a language requires persistent accumulation and practice. The huge differences between Chinese and English make it more difficult to understand and learn. Vocabulary memory and grammar understanding are of great significance for more complex English expression in the later stage. For students with weak foundation or little interest in English, college English learning will be more difficult.

\subsection{Lack of Sufficient Interaction Between Teachers and Students}

In school education, due to the arrangement of class hours and the large number of classes, teachers can not take care of the learning situation of each student. They can only proceed according to the general direction of the course and the learning progress of most students. As students, they need to try to keep up with the teacher's progress. They have little time to stop and think about the point and exchange their understanding of knowledge with the teacher.

\subsection{Lack of Interest in Learning}

The reasons why students are not interested in learning can be divided into two categories: 
Academic performance has an impact on confidence. In the same class, students' acceptance of knowledge is different. In addition to each student's early basic knowledge, learning efficiency and interest, it is also closely related to students' preview of class. However, many students do not pay attention to this link. The main reason is that they have no interest to the single written text, and lack of selfconsciousness to learn actively. Therefore, after a period of study, gaps will gradually form, causing students to lose the confidence and interest in continuing to study.

The single teaching method. Since ancient times, teachers have been in a leading position in the classroom, teaching the contents to students step by step, and explaining knowledge in their mother language. Although it is easy for students to understand, in the long run, this kind of atmosphere is not conducive to cultivating students' English language sense and foreign language thinking, nor can it internalize and apply knowledge again. In this way, teachers will feel tired in the teaching process with low teaching efficiency.

\section{APPLICATION \& ADVANTAGES OF SHORT VIDEO IN THE CLASSROOM}

The transmission mode of short video mainly involves two modality of vision and hearing, including text, pictures, sounds and actions. While rapidly transmitting information, it will also create a relaxed and pleasant atmosphere. College students' curiosity about new things can fully stimulate their learning enthusiasm. Therefore, we can combine their characteristics to discuss a new teaching mode.

\subsection{Specific Application of Short Video in Teaching}

\subsubsection{Assist outside the classroom}

One of the ways of effective learning is pre-class preview and after-class review. To this end, teachers can find some enlightening videos for students to watch in advance, and guide them to preview textbooks or find materials to solve problems through the questions in the videos. The review after class is conducive to students' mastery of the completeness of knowledge, so the teacher can sort out the content of this lesson and present it with video to complete the entire learning process.

The ultimate goal of foreign language learning is to use it. You can't just study it with a book. It will be more memorable in practical situations. This requires teachers to learn to change their roles and provide students with opportunities for language practice, so that they can become the senders of auditory and visual modes, not always the receivers[6]. For example, organizing students to carry out dubbing activities and drama performances of classic dramas can effectively train students' audio-visual and oral skills. The repetition of knowledge in the preparation process will deepen their understanding of vocabulary use, stimulate students' creativity in other aspects and find characteristics other than their learning.

\subsubsection{Apply to the classroom}

PowerPoint (PPT) courseware is a widely used medium in modern teaching. It not only meets the requirements of multi-modality which is conducive to students' overall grasp of what they are learning, but its operation and requirements are simple.

The PPT courseware supports hyperlinks, and teachers can put the short videos they need in the form of hyperlinks, which is simple and convenient. On the one hand, students need to learn to follow the audio and speak English. They can actually participate in it and cultivate their sense of language through continuous reading. On the other hand, we also need to feel the atmosphere of foreign language communication through pure foreign language audio, exercise foreign language thinking, and experience the application of words in practical application situations and common sentence expressions, so as to consolidate what we have learned. The video contains animation, pictures and sounds, which can mobilize people's vision and hearing at the same time and make learning no longer boring. This method not only provides an atmosphere for students, but also builds a multi-modal environment for teaching.

In social communication, when one modality is not enough to express the meaning of the communicator, people will use another modality to strengthen, supplement, adjust and cooperate, so as to achieve a more full expression of the meaning and let the listener understand the discourse[6] The various modalities in short video can give full play to this advantage. In the whole process of teaching interaction, oral expression is the main modality, and the modality in courseware plays an auxiliary role. In addition, teachers can also use actions, gestures, expressions and tones to attract students' attention and emphasize important contents. If necessary, teachers can use teaching aids to make abstract contents concrete and reduce students difficulties in understanding.

\subsubsection{Strengthen in Exercises}

In the large class education in schools, teachers often only explain difficult or representative exercises in class because of time and course progress. However, there are many students do not master knowledge. Some simple exercises are also very difficult for them, so the short videos is very necessary. The teacher can share the video explaining exercises to the learning group or personal account, so that students can watch it repeatedly and solve their problems in time. They will not delay the problems 
again and again, but improve their learning confidence step by step.

\subsection{Advantages of Using Short Video Teaching}

Short time and little content. The duration of the video is generally 15 seconds to a few minutes with brief and clear content. It is in line with the characteristics of the public receiving information quickly. On the other hand, the length of the video also determines that the information content will not be too much and complex. In terms of the information content, it forms a sharp contrast with the knowledge in the whole class. At the same time, there is no seriousness in classroom teaching, and students can face learning in a more relaxed mood.

Free learning time and place. The time and place of the classroom in the school have been arranged. For the lecture content in the teacher's classroom, students should try their best to keep up with the teacher's progress and mark it for review after class. Because the teacher can't have time to do it again, while the recorded video supports saving and replaying. This learning method appropriately complements the shortcomings of classroom teaching and plays an auxiliary role in classroom teaching.

Wide range of transmission of short video. Whether it is uploaded to a personal social account or communicated in a learning group, the video supports the sharing function. If it is in the account, it will also have the comment function. Students can ask questions to the teacher about the knowledge points in the video, and everyone they see can participate in the discussion. The process of discussion is an exchange and consolidation of knowledge.

High efficiency. The traditional teaching method is the combination of books, chalk and blackboard, which is a single teaching method. While the short video is short but more inclusive, it can convey more information and make full use of network resources to explain obscure knowledge.

It is conducive to the analysis of teaching situation[7].

Teachers can collect and analyze according to the background video views and the focus of students' discussion, so as to find students' common learning difficulties, and then adjust the teaching emphasis. Such data analysis can enable teachers to explain difficult contents to students, provide corresponding guidance, and finally improve students' learning effect and teachers' teaching effect.

\section{CONCLUSION}

The emergence of short video not only brings people new ways to obtain information and user experience, but also triggered our new thinking. Based on the analysis of the rapid development of short video, this article discusses the application of short video in College English multi-modal teaching, which should not only consider the characteristics of the discipline, but also pay attention to the personalized characteristics of College Students' physical and mental development. The multi-modal short video teaching mode can mobilize students' senses in the learning process, focus on learning content, reduce students' dull learning burden and strengthen their language application ability. In the teaching process, teachers should not only play their leading role and pay attention to students' subjective initiative, but also take practical teaching models to improve their enthusiasm to participate in language use.

\section{REFERENCES}

[1]Zhang Delu. 2009. Application of Multi-modal Discourse Theory and Media Technology in Foreign Language Teaching [J]. Foreign Language Teaching (4):15-20. (In Chinese)

[2]Levine, P. \& Scollon, R.. 2004. Discourse and Technology: Multimodal Discourse[C]. Washington D.C.: Georgetown University Press.

[3]Kress, G. \& Van Leeuwen. 1996. Reading images[M]. London: London Routledge

[4]China Internet Network Information Center (CNNIC).The 46th Statistical Report on China's Internet Development [EB/OL]. (In Chinese) DOI: http://www.gov.cn/xinwen/202009/29/content_5548176.htm

[5]Xinhuanet.com. Tiktok Data Report [EB/OL].2020. (In Chinese) http://www.xinhuanet.com/tech/202101/05/c_1126948875.htm

[6]Zhang Delu, Wang Lu. 2010. Synergy of Multimodal Discourse Modality and its Embodiment in Foreign Language Teaching [J]. Foreign language journal, (2):97-102. (In Chinese)

[7]Liu Mei. Teaching and Research on English Writing in the Era of Big Data [M]. Jiangsu: Soochow University Press, 2019. (In Chinese) 